\title{
Susceptibilidad genética frente al cáncer bucal por infección del virus del papilloma humano
}

\author{
Martha Rebolledo-Cobos ${ }^{1}$ y Zuleima Yáñez-Torregroza² \\ ${ }^{1}$ Universidad Metropolitana, Programa de Odontología, Atlántico, Colombia; ²Universidad Simón Bolívar, Facultad de Medicina, Ciencias Básicas \\ y Biomédicas, Atlántico, Colombia
}

\section{Resumen}

Los carcinógenos ambientales y el virus del papiloma humano (VPH) son los principales responsables del cáncer bucal. Los factores de susceptibilidad en el genoma humano desempeñan un papel modulador del riesgo, sin embargo, no todos los individuos expuestos a los carcinógenos padecen cáncer. El objetivo de la presente revisión es describir los principales factores de susceptibilidad genética para cáncer bucal en individuos con infección por VPH. Se realizó una búsqueda sistemática en tres bases de datos en inglés; solo siete artículos cumplieron con los criterios de selección. Se registraron polimorfismos genéticos en tres categorías relacionados con el VPH y que participan en la oncogénesis. Se identificaron tres artículos relativos a la desregulación de los mecanismos de control del ciclo celular, uno relativo a mutaciones en la vía de la apoptosis y tres a polimorfismos en genes de respuesta inflamatoria e inmune. La asociación entre polimorfismos para el desarrollo de cáncer bucal y VPH es evidente, aunque continúa en estudio ya que no siempre el patrón de oncogénesis de las neoplasias bucales está relacionado con el VPH, sino con otros factores ambientales o epigenéticos.

PALABRAS CLAVE: Susceptibilidad genética. Cáncer bucal. Polimorfismos. Virus del papiloma humano. Mutación.

\begin{abstract}
Environmental carcinogens and human papillomavirus (HPV) are the main responsible factors for oral cancer. Susceptibility factors in the human genome play a risk-modulating role; however not all individuals exposed to these carcinogens suffer from cancer. The purpose of the present review is to describe the main factors of genetic susceptibility to oral cancer due to HPV infection. A systematic search was carried out in three databases in English, with only 7 articles meeting the selection criteria. Genetic polymorphisms are shown in three categories, which are related to HPV and participate in oncogenesis. Three articles related to deregulation of cell cycle control mechanisms were identified, as well as one referring to mutations in the apoptosis pathway and three about polymorphisms in inflammatory and immune response genes. The association of polymorphisms for the development of oral cancer by HPV is evident, although it remains under study. Oral neoplasms' oncogenesis pattern is not always associated with HPV, but with other environmental or epigenetic factors.
\end{abstract}

KEY WORDS: Genetic susceptibility. Oral cancer. Polymorphisms. Human papillomavirus. Mutation.

Fecha de recepción: 17-05-2018

Fecha de aceptación: 28-08-2018

DOI: 10.24875/GMM.18004408
Gac Med Mex. 2019;155:284-290

Disponible en PubMed www.gacetamedicademexico.com 


\section{Introducción}

La descripción de las variantes en las secuencias del ADN humano y las secuencias de proteínas iniciaron con dos publicaciones independientes realizadas por Beaudet et al. y Beutler et al. en 1993. Esos autores propusieron que cualquier cambio anormal en la secuencia de los nucleótidos habitualmente es una mutación, ${ }^{1,2}$ que puede o no causar cambios fenotípicos en los individuos afectados y no necesariamente corresponde a una enfermedad y puede heredarse de los padres (mutación en la línea germinal) o adquirirse y manifestarse a lo largo de la vida de un individuo (mutación somática o multifactorial).

En este último tipo de mutación existe específicamente una variación en la secuencia de ADN que puede ocurrir en una población con una frecuencia de $1 \%$ o superior, denominada polimorfismo. ${ }^{3}$ Los polimorfismos ocurren naturalmente, con un efecto neutral, beneficioso o promotor de enfermedad; también pueden ser de uno o más cambios de nucleótidos, al igual que otras mutaciones. El polimorfismo de un solo nucleótido es el más común, surge de cada 1000 pares de bases (pb) en el genoma humano y generalmente se encuentra en áreas que flanquean genes codificadores de proteínas o regiones reconocidas como críticas para la unión de microARN y regulación de expresión de genes/proteínas. ${ }^{3}$ Sin embargo, los polimorfismos de un solo nucleótido también pueden ocurrir en secuencias codificantes, intrones o regiones intergénicas. ${ }^{4}$

En el contexto de las enfermedades hereditarias y las multifactoriales, una mutación ha sido definida como una alteración en la secuencia del gen que anula su función biológica; es posible también encontrarla en tumores malignos de la cavidad bucal cuando el individuo se encuentra expuesto a ciertos virus o factores de riesgo epigenéticos. ${ }^{5}$ En general son pocos los tipos de cáncer para los cuales se han podido identificar uno o más genes cuyas mutaciones conceden un alto riesgo para presentarlos. En cuanto al cáncer bucal (CB), en especial el carcinoma escamocelular y otros cánceres de cabeza y cuello, si bien es cierto que presentan múltiples etiologías y factores de riesgo adquiridos como el consumo de alcohol, cigarrillo o tabaquismo, enfermedades de transmisión sexual o infecciones por virus de inmunodeficiencia humana, herpes simple o papiloma humano (VPH), ${ }^{6,7}$ En este último caso se identifican mutaciones de diferente índole que otorgan susceptibilidad a enfermedades neoplásicas a partir de una primoinfección viral en algún momento de la vida. ${ }^{4-7}$ El VPH es causante de otras lesiones neoplásicas que a veces persisten, pero no necesariamente evolucionan a cáncer, lo que se relaciona con sus genotipos: de alto riesgo (hrHPV) y bajo riesgo (IrVPH); los más asociados con CB son los genotipos 16, 18, 31 y 45 , considerados de alto riesgo. ${ }^{6-8}$

Existen investigaciones que relacionan el componente somático en la aparición del CB atribuido a hrVPH por cambios en los genes involucrados con el ciclo celular (desempeñan un papel en la modulación de la reparación del ADN celular), el control del ciclo celular, el crecimiento celular, la apoptosis y la respuesta inflamatoria e inmune, los cuales en condiciones fisiológicas suelen dar estabilidad al genoma humano. ${ }^{8-14}$ La presente revisión sistemática tiene como objetivo describir los principales factores de susceptibilidad genética para el desarrollo de CB o carcinoma escamocelular frente a infección por VPH.

\section{Método}

- Población: Pacientes infectados con VPH y que desarrollaron CB.

- Intervenciones: Diagnóstico molecular de mutaciones y genotipificación del VPH en relación con la presencia de CB.

- Objetivo: Identificar factores de susceptibilidad frente al CB por la infección del VPH.

- Estudios incluidos: estudios de casos y controles, revisión sistemática o metaanálisis.

\section{Criterios de inclusión}

- Artículos en inglés en texto completo.

- Estudios publicados entre el 1 de enero de 2013 y el 31 de enero de 2018.

- Estudios que evaluaban la susceptibilidad genética a CB debido a infección por VPH.

- Estudios que brindaran información sobre polimorfismos de control del ciclo celular en el $C B$ por VPH.

- Estudios que proporcionaran información sobre polimorfismos por variaciones en la apoptosis.

- Estudios que brindaran información sobre polimorfismos de genes de respuesta inflamatoria e inmune.

\section{Criterios de exclusión}

- Estudios que proporcionaran información inadecuada. 


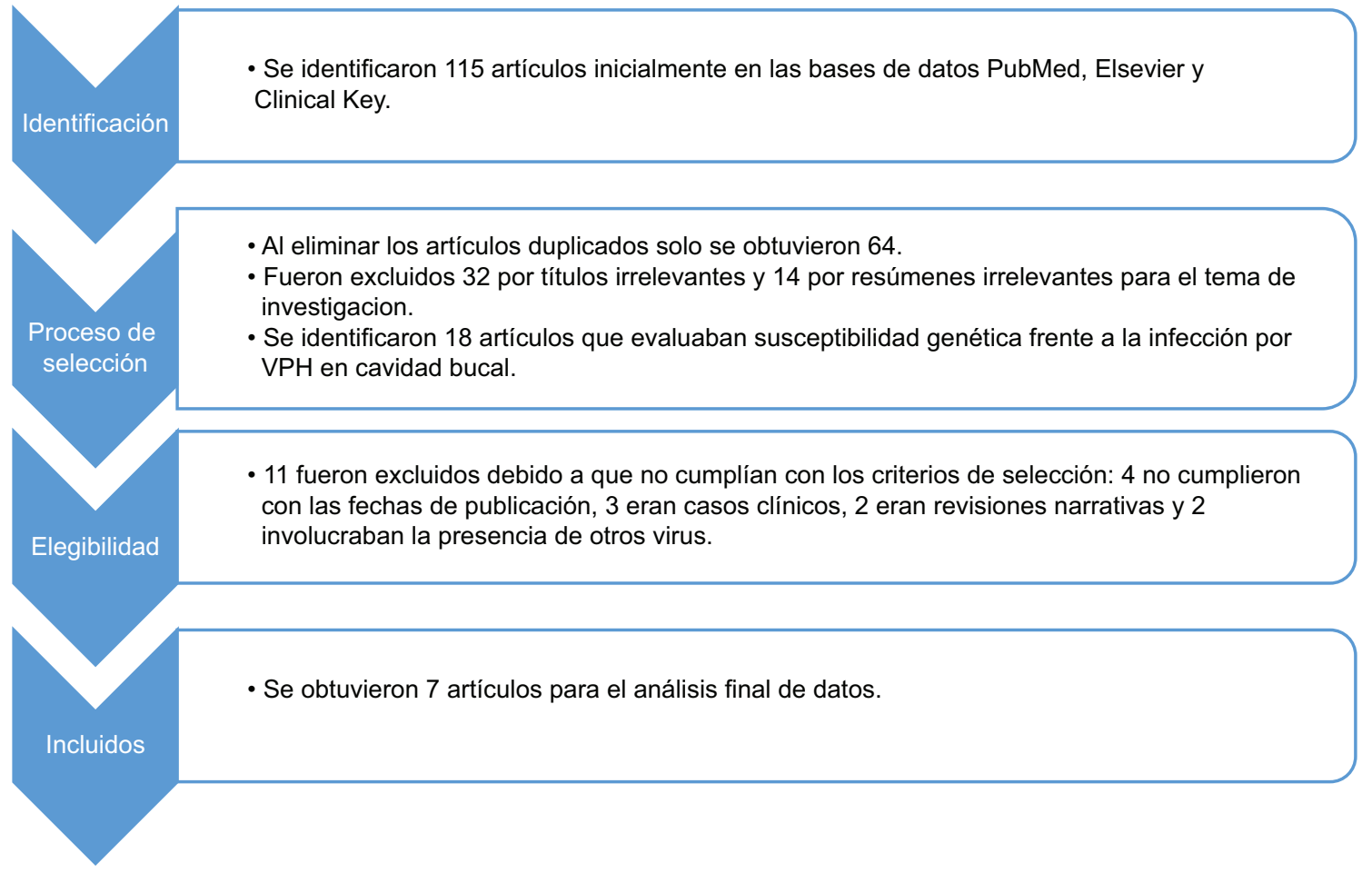

Figura 1. Diagrama de flujo del proceso de selección de los artículos revisados.

- Revisiones narrativas.

- Estudios in vitro.

- Estudios en animales.

- Estudios que involucraban alteraciones potencialmente malignas.

- Estudios que incluían otras infecciones, como por ejemplo por virus de inmunodeficiencia humana o herpes simple.

- Estudios donde no se hubiera realizado diagnóstico molecular y genotipificación.

\section{Fuentes de información y búsqueda}

Se realizó una extensa búsqueda sistemática de literatura de forma manual, empleando las bases de datos PubMed, Elsevier y Clinical Key, utilizando la combinación de los siguientes descriptores: HPV and oral cancer, human susceptibility, HPV infection, oral cavity, polymorphisms of a single nucleotide, polymorphisms of cell cycle control, variations in apoptosis and polymorphisms of inflammatory and immune response genes. Los artículos involucraron solo investigaciones en humanos, sin distinción de sexo ni edad (Figura 1).

\section{Selección de estudios}

Inicialmente se registraron 115 artículos en una hoja de cálculo electrónica. Después de eliminar los artículos duplicados, solo se consideraron relevante 64 por sus títulos y resúmenes; se identificaron 18 que evaluaban susceptibilidad frente a la infección por VPH en cavidad bucal, de los cuales 11 fueron excluidos debido a que no cumplían con los criterios de selección. En el análisis final solo se incluyeron siete artículos (Figura 1).

\section{Recopilación de datos}

Se proporcionó un instrumento tipo formato piloto estándar para la extracción de datos por parte del experto; solo se tuvieron en cuenta los elementos apropiados para la presente revisión. El experto verificó y aprobó los datos, los cuales se extrajeron y se tabularon en una hoja de cálculo electrónica de Excel, una por cada artículo seleccionado.

De los siete artículos, tres hacían referencia a polimorfismos del control del ciclo celular, uno a polimorfismos por variaciones en la apoptosis y tres a polimorfismos de los genes de respuesta inflamatoria e inmune (Figura 1).

Los datos de interés fueron los siguientes: nombre del artículo, nombre del autor, país, año de publicación, diseño del estudio, tamaño de muestra, configuración, población, genotipificación, tipo de polimorfismo/mutación, tipo de cáncer, presencia de la infección por VPH, factores de riesgo o intervención de comparación, nivel de riesgo, resultados y observaciones. 


\section{Resultados}

Para que la infección por hrVPH persista y se extrapole a la formación de cáncer, existen factores genéticos del huésped que contribuyen en la variabilidad en que se desarrollan estas neoplasias malignas posterior a la infección viral. ${ }^{7}$ Las oncoproteínas virales E6 y E7 del VPH desactivan los genes supresores de tumores p53 y pRb, permitiendo a la célula escapar de los puntos de control del ciclo celular normal, con la consiguiente transformación celular, inmortalización y oncogénesis. ${ }^{7}$ Diversos autores señalan tres vías simultáneas para el análisis de polimorfismos genéticos que pueden ayudar a comprender los distintos mecanismos implicados en la aparición de $C B$ y carcinoma escamocelular asociados con el VPH: polimorfismos de control del ciclo celular, variaciones en la apoptosis y polimorfismos de genes de respuesta inflamatoria e inmune. ${ }^{7}$

\section{Polimorfismos de control del ciclo celular}

El primer tipo de polimorfismo corresponde a las interacciones entre el genoma humano y el genoma del hrVPH; el mecanismo de acción radica en la relación entre el gen supresor tumoral p53 y la oncoproteína E6 del VPH. Esta proteína no estructural se vincula al gen p53 e inicia su degradación, lo que ocasiona proliferación celular descontrolada. ${ }^{7,8} \mathrm{El}$ gen p53 está localizado en el brazo corto del cromosoma 17, banda 13 (17p13.1), tiene aproximadamente $20 \mathrm{~kb}$ y consta de 11 exones, el primero no codificante y colocado entre 8 y $10 \mathrm{~kb}$ de los exones 2 a 11 . El $90 \%$ de este tipo de mutaciones se han localizado en los exones 5 a 8 del gen; aproximadamente 20 a $30 \%$ de las mutaciones se produce en los cinco codones hot-spot que se encuentran en estos exones.

Otras alteraciones de p53 son deleciones, inserciones, mutaciones en los lugares de splicing y pérdidas de heterocigosidad. ${ }^{7,8}$ El mecanismo más común de pérdida de funcionalidad de p53 es la mutación puntual de uno de los alelos y deleción del otro. En este sentido, cabe destacar que algunas mutaciones de p53 son dominantes, lo que constituye una excepción a la norma de que los genes supresores manifiestan su acción oncogénica solo si se produce una alteración de ambas copias del gen; ${ }^{9,10}$ un solo monómero proteico defectuoso procedente de la mutación de uno de los dos alelos del gen p53 es suficiente para provocar la inactivación total de la proteína tetramérica. Cuando los monómeros mutados forman complejos con los monómeros normales se forma una proteína p53 mutante cuya vida media se alarga sensiblemente. ${ }^{7,8}$

\section{Variaciones en la apoptosis}

Durante la carcinogénesis, los tumores tienen que desarrollar múltiples mecanismos para superar la vigilancia inmune del huésped y la apoptosis intrínseca 0 el arresto del ciclo celular. La diferencia individual en la resistencia a la apoptosis a través de la vía FAS podría permitir que muchos cánceres escapen al sistema inmune o lo contraataquen. Las variantes de la línea germinal en las vías extrínseca e intrínseca podrían afectar la eficacia apoptótica y la resistencia a la apoptosis y, en consecuencia, influir en la infección por VPH. ${ }^{7,11}$ Lo anterior puede ser particularmente relevante para un mecanismo viral que funciona a través del ciclo celular y mecanismos apoptóticos. Se ha sugerido que los polimorfismos genéticos de los promotores FAS y FASL contribuyen al riesgo de cáncer asociado con el VPH, induciendo la apoptosis diferencial de las células inmunes en respuesta a las señales microambientales después de la infección por VPH. Se ha descubierto que el polimorfismo en la posición 670 del promotor FAS suprime el sitio de unión para el elemento de transcripción nuclear y altera la expresión del gen FAS; el homocigoto pro 72 del polimorfismo p53 en el codón 72 parece ser un importante regulador de la apoptosis a través de la vía FAS/FASL en cáncer de cabeza y cuello. ${ }^{11}$

\section{Polimorfismos de genes de respuesta inflamatoria e inmune}

Mientras que los hrVPH se entienden bien como factores de riesgo para CB y cáncer de cabeza y cuello, la investigación de factores genéticos del huésped en las respuestas inflamatorias e inmunes a la infección por VPH podría ayudar a comprender la asociación entre la infección por VPH y el CB. Son precisamente estas respuestas o la eficacia de la vigilancia inmune lo que modifica la eliminación del VPH o la infección persistente. ${ }^{7,12}$ La inflamación es una parte de la respuesta del huésped a estímulos ambientales internos o externos, que se promueve por la acción de citocinas proinflamatorias, incluidas las interleucinas (IL) 1 y 6 , factor de necrosis tumoral e interferón- $\gamma$, y se resuelve comúnmente con 
antiinflamatorios. Las citocinas IL-4, IL-10, IL-13, así como el interferón- $\alpha$ y el factor de crecimiento transformante- $\beta$ desempeñan un papel en el control del crecimiento de células infectadas por el VPH. La persistencia viral, la progresión de la enfermedad o la transformación a cáncer implican escapar de estos mecanismos. Por lo tanto, las variantes de la línea germinal de estas citocinas podrían modificar la eficacia de la defensa contra el VPH y, por consiguiente, las tasas de infección. ${ }^{12}$

El éxito del VPH para disminuir las respuestas inmunes puede ser importante en la patogénesis del cáncer asociado con el VPH, ya que la etiología del cáncer inducido por el VPH desencadena una infección viral persistente que puede ser minimizada por una respuesta inmune efectiva. Los polimorfismos de varios genes de citocinas se han implicado en la inducción de susceptibilidad o resistencia a cánceres causados por la infección por VPH debido a su papel en la determinación de la respuesta inmune del huésped. Por lo tanto, las variantes genéticas de genes de citocinas en regiones promotoras o codificadoras que se cree que influyen en los niveles de expresión o la eficacia funcional pueden estar implicadas en la susceptibilidad al estado de VPH de pacientes con carcinoma escamocelular y $\mathrm{CB} .13,14$

Algunos estudios han relacionado polimorfismos en genes de citocinas proinflamatorias y antiinflamatorias, particularmente en regiones reguladoras, con variaciones intraindividuales en la producción de citocinas y el riesgo de cáncer. La asociación de polimorfismos de citocinas de interferón- $\gamma$ e IL-10 con el riesgo de cáncer ha sido bien documentada: en pacientes con cáncer cervical positivos para VPH se ha observado transcripción de interferón- $\gamma$ disminuida y transcripción de IL-10 aumentada. ${ }^{12,14}$ El interferón- $\gamma$ desempeña un papel fundamental en la defensa contra virus y patógenos intracelulares mediante la inducción de respuestas inflamatorias mediadas inmunes. El polimorfismo de un solo nucleótido $\mathrm{T}+874 \mathrm{~A}$ localizado en el sitio de inicio de la traducción del gen del interferón- $\gamma$, que coincide con un sitio de unión del factor de transcripción nuclear kappa B putativo, podría ser fundamental en la inducción de una producción constitutivamente alta de interferón- $\gamma$. Los +874 alelos $T$ a $A$ con bajo (AA), medio (AT) y alto (TT) también se han asociado significativamente con la producción de citocinas. La IL-10 tiene un efecto supresor sobre la inmunidad mediada por células, que puede ser crítica en la eliminación de los QT que hospedan el VPHg. ${ }^{13}$ Existe diversos polimorfismos en el gen IL-10, entre los cuales el de un solo nucleótido en la posición 1082 de la región promotora desempeña un papel importante en la determinación de producción alta, media y baja de IL-10.

La asociación de G/A polimorfismo de un solo nucleótido en la posición 1082 se ha asociado con producción baja $A A$, media $A G$ y alta $G G$ de citocinas. También se ha informado que los polimorfismos en los genes humanos de IL-1b y factor de necrosis tumoral- $\alpha$ influyen en la expresión de citocinas y factor de crecimiento transformante, que regula la proliferación y apoptosis de las células epiteliales. ${ }^{7,13,14} \mathrm{El}$ factor de necrosis tumoral- $\alpha$ puede controlar directamente la infección por VPH por inducción de apoptosis en células infectadas por VPH, como las células de cáncer de cuello uterino y CB. La perturbación del equilibrio entre los niveles de citocinas pro y antiinflamatorias puede ser causada por mutaciones genéticas heredadas, a partir de las cuales las variantes genéticas comunes también pueden modificar la expresión o función de genes clave, trastornando el equilibrio de las citocinas y afectando el riesgo y resultado del cáncer. ${ }^{13,14}$

Las investigaciones consultadas para la síntesis de esta revisión muestran la biotransformación, desintoxicación, eliminación o control inmune de carcinógenos como el VPH, junto con los mecanismos de regulación celular, reparación del ADN y las vías apoptóticas, como estrategias intrínsecas de evadir el cáncer. ${ }^{7}$ Tandon et al. y Saleen et al. describen polimorfismos en el gen p53 localizado en el brazo corto del cromosoma 17, banda 13 (17p13.1). Por su parte, Murali et al. los relacionan con el gen p27 y consideran que contribuyen en la patogénesis del CB. Solo se encontraron tres artículos relativos a este tipo de polimorfismo, dos de la India y uno de Pakistán ${ }^{10}$ (Tabla 1).

En un solo artículo se evidenció que existen mecanismos para superar la vigilancia inmune del huésped y la apoptosis intrínseca. Sun et al. consideran que las variantes del promotor FAS/FASL en las vías de la apoptosis alteran la actividad transcripcional de esos genes y la regulación de la muerte celular. Sin embargo, manifiestan que ningún estudio ha investigado si los sitios tumorales contribuyen a la asociación entre los polimorfismos FAS/FASL y el riesgo de una recurrencia tumoral. ${ }^{11}$ También identificaron que los pacientes con índice de genotipo para cáncer orofaríngeo y FASL $844 \mathrm{CT} / \mathrm{TT}$ tenían un riesgo significativamente mayor de recurrencia del cáncer $(\mathrm{cHR}=2.5, \mathrm{IC} 95 \%=1.1-5.8, \mathrm{p}=0.043 ; \mathrm{HR}=2.7$, IC $95 \%=1.2-6.0, p=0.032$ ) en comparación con los 
Tabla 1. Polimorfismos de control del ciclo celular por virus del papiloma humano en cáncer bucal y carcinoma escamocelular

\begin{tabular}{|c|c|c|c|c|c|c|c|}
\hline Polimorfismo & Mutación & Frecuencia del genotipo & $n$ & $p$ & $\begin{array}{l}\text { Diagnóstico } \\
\text { oncológico }\end{array}$ & País & Autor \\
\hline \multirow[t]{3}{*}{$\begin{array}{l}\text { Vía de control } \\
\text { del ciclo } \\
\text { celular }\end{array}$} & Codón 72 de p53 & $\begin{array}{l}\text { Arg/Arg }=23 \%(8) \\
\text { Arg/Pro }=57 \%(20) \\
\text { Pro/Pro }=20 \%(7)\end{array}$ & 35 & $<0.809$ & CEC & India & Tandon et al..$^{8}$ \\
\hline & Codón 72 de p53 & $\begin{array}{l}\text { Arg }) / \operatorname{Arg}=8.47 \%(22) \\
\text { Arg/Pro }=43.46 \%(113) \\
\text { Pro/Pro }=4807 \%(125)\end{array}$ & 260 & $<0.001$ & CEC & Pakistán & Saleem et al. ${ }^{9}$ \\
\hline & p27 & $\begin{array}{l}\text { p27 (rs34329; } \\
\text { relación 3.05, IC } 95 \% \text { \% 2.12-4.40). } \\
\text { ciclina E (rs1406), ciclina H (rs3093816), } \\
\text { ciclina D1-1 (rs647451), ciclina } \\
\text { D2 (rs3217901) y Rb1-2 (rs3092904). }\end{array}$ & $6 / 9$ & $<0.0001$ & $\mathrm{CB}$ & India & Murali et al. ${ }^{10}$ \\
\hline
\end{tabular}

$\mathrm{CEC}=$ carcinoma escamocelular, $\mathrm{CB}=$ cáncer bucal

Tabla 2. Polimorfismo de la vía de la apoptosis ${ }^{11}$

\begin{tabular}{|l|l|c|c|}
\hline Via & Genotipo & n & $\%$ \\
\hline FAS (mutación) & & & \\
\hline FAS 670 A > G & AA & 7 & 16.7 \\
\hline FAS 1377 G > A & AG + GG & 35 & 83.3 \\
& GG & 34 & 80.9 \\
\hline FASL (mutación) & AG+AA & 8 & 19.1 \\
\hline FASL 124 A > G & AA & & \\
\hline FASL 844 C > T & AG + GG & 11 & 73.8 \\
& CC & 11 & 26.2 \\
\hline & CT + TT & 31 & 73.8 \\
\hline
\end{tabular}

pacientes con genotipo FASL 844 CC como grupo de referencia, mientras que los pacientes sin cáncer orofaríngeo con genotipos FAS 670 AG/GG y FASL $844 \mathrm{CT} / \mathrm{TT}$ tuvieron un riesgo significativamente mayor que los pacientes con riesgo de recurrencia tumoral $(\mathrm{cHR}=2.2$ y $1.8, \mathrm{IC} 95 \%=1.2-5.7$ y $1.1-3.2$, $\mathrm{p}=0.04$ y 0.041 , respectivamente; $\mathrm{aHR}=2.4$ y 1.7 , IC $95 \%=1.1-5.1$ y $1.0-3.0, p=0.043$ y 0.049 , respectivamente) en comparación con sus correspondientes genotipos AA y $\mathrm{CC}^{11}$ (Tabla 2).

En las investigaciones consultadas, los hrVPH estuvieron ampliamente descritos como factores de riesgo para cáncer de cabeza y cuello y CB. El huésped ejecuta respuestas ineficaces en la vigilancia inmune frente a infecciones por VPH, lo que provoca que se perpetúe la presencia viral y la transformación maligna; al respecto se hallaron tres artículos.

Jin et al. encontraron que la seropositividad al VPH 16 únicamente se asoció con aumento del riesgo de $\mathrm{CB}(\mathrm{RM}=3.1, \mathrm{IC} 95 \%=2.1-4.6)$ y que el riesgo de cáncer orofaríngeo asociado con VPH 16 se modificó por cada polimorfismo de un solo nucleótido. ${ }^{12}$ Además, observaron resultados similares para los genotipos de riesgo combinados de cuatro variantes y todas estas asociaciones significativas fueron más pronunciadas en varios subgrupos, particularmente en pacientes con cáncer orofaríngeo y que nunca fumaron. ${ }^{12}$

Por su parte, Hsu et al. manifestaron que existen dos citocinas involucradas en la carcinogénesis. ${ }^{13} \mathrm{Ob}$ servaron asociación entre el polimorfismo en TGF- $\beta 1$ (polimorfismo $\mathrm{G}$ a $\mathrm{C}$ en el codón $25<+915>$ ), IL-10 (1082 G/A, 819 C/T y 592 C/A) y riesgo de CB en 162 pacientes. Encontraron que el codón del genotipo 25 GC de TGF- $\beta 1$ es significativamente más frecuente en pacientes con $\mathrm{CB}$ en comparación con un grupo control sano $(p<0.0001)$. Los pacientes con genotipo $\mathrm{GC}$ en el codón 25 tenían un riesgo 11.09 veces mayor de CB (RM = 11.09, IC $95 \%=6.16-113.23)$. Evidenciaron polimorfismos de IL-10 en posiciones 819 y 592 correlacionados con el riesgo de CB $(p<0.0001)$. Los genotipos que presentaron alelos IL-10-592 C tenían un mayor riesgo de $\mathrm{CB}(\mathrm{RM}=1.79$, IC $95 \%=1.11-2.91)$. Los pacientes con el genotipo de TC en IL-10-819 tenían 3.32 mayor riesgo de CB $(\mathrm{RM}=3.32$, IC $95 \%=1.64-6.94) .{ }^{13}$

Antonsson et al. sugieren que los polimorfismos en los dos genes EV (EVER1 y EVER2, también conocidos como proteínas del canal transmembrana 6 y 8 ) están involucrados, ya que un pequeño número de mutaciones críticas en estos genes son causa de infecciones por VPH y formación de cáncer. Estos autores no evidenciaron ninguna asociación con el estado de los polimorfismos de un solo nucleótido EVER1/EVER2 y el estado del VPH, ni tampoco con la presencia de $\mathrm{CB}^{14}$ (Tabla 3).

\section{Conclusiones}

Los estudios consultados en la presente revisión muestran amplia evidencia de la relación entre la 
Tabla 3. Polimorfismos de genes de respuesta inflamatoria e inmune

\begin{tabular}{|c|c|c|c|c|c|c|}
\hline Polimorfismo & $\mathrm{n}$ & Factor de riesgo & Genotipo VPH & Riesgo & Cáncer & Autor \\
\hline $\begin{array}{l}\text { Polimorfismo de un solo nucleótido } \\
\text { TNF- } \alpha \\
\text { [-308G > A (rs1800629), } \\
-857 C>\text { T (rs1799724), } \\
-863 C>A(\text { rs1800630) y } \\
-1031 T>C \text { ( rs1799964)] }\end{array}$ & $176 / 325$ & $\begin{array}{l}\text { - Consumo tabaco } \\
\text { - Consumo alcohol } \\
\text { - Edad } \\
\text { - Sexo }\end{array}$ & 16 hrVPH & $\begin{array}{c}\mathrm{RM}=3.1 \\
\mathrm{IC} 95 \%=2.1-4.6\end{array}$ & $\mathrm{CCC}$ & Jin et al..$^{12}$ \\
\hline $\begin{array}{l}\text { TGF-b1 (G a C } \\
\text { polimorfismo en el codón } 25 \\
<\text { p915>) e IL-10 (1082 G/A, } \\
-819 \text { C/T y } 592 \text { C/A) }\end{array}$ & 161 & $\begin{array}{l}\text { - Consumo tabaco } \\
\text { - Consumo alcohol } \\
\text { - Edad } \\
\text { - Sexo } \\
\text { - Clasificación y } \\
\text { ubicación tumoral }\end{array}$ & $\mathrm{VPH}+$ & $\begin{array}{c}\mathrm{RM}=11.09 \\
\text { IC } 95 \%=6.16-113.23\end{array}$ & $\mathrm{CB}$ & Hsu et al. ${ }^{13}$ \\
\hline $\begin{array}{l}\text { Polimorfismo de un solo nucleótido } \\
\text { EVER } 1 \text { (rs2613516, rs12449858) y } \\
\text { EVER } 2 \text { (rs7205422, rs12452890) }\end{array}$ & $0 / 219$ & Ninguno & $\mathrm{VPH}+$ & 0 & $\mathrm{CCC}$ & Antonsson et al. ${ }^{14}$ \\
\hline
\end{tabular}

$\mathrm{CCC}=$ cáncer de cabeza y cuello, $\mathrm{CEC}=$ carcinoma escamocelular.

infección por VPH y la presencia de CB, aparte de los factores de riesgo epigenéticos, como consumo de tabaco y alcohol, la edad y relaciones sexuales modificadas. La integración viral en el genoma celular de la mucosa bucal hace susceptible genéticamente al ser humano a desarrollar tumores malignos. Se ha observado la presencia de polimorfismos genéticos de tres categorías y mecanismos de participación simultáneos en el proceso de oncogénesis, así como la identificación de genes o oncoproteínas transformantes que inducen desregulación de los mecanismos de control del ciclo celular, produciendo inestabilidad en el genoma, alteración de la apoptosis e inestabilidad en los genes de respuesta inflamatoria e inmunológica.

El estudio de la asociación de estos polimorfismos para el desarrollo de CB frente a la infección por VPH aún se encuentra en desarrollo; además, no siempre el patrón de oncogénesis de todos los cánceres de cabeza y cuello o CB se encuentra relacionado solo con la presencia del VPH, sino con diversos factores de riesgo medioambientales o hereditarios. En el contexto colombiano no se encontraron estudios exhaustivos que hicieran énfasis en la susceptibilidad genética humana frente al $\mathrm{CB}$, carcinoma escamocelular o cáncer de cabeza y cuello por la infección del $\mathrm{VPH}$.

\section{Bibliografía}

1. Beaudet AL, Tsui LC. A suggested nomenclature for designating mutations. Hum Mutat. 1993;2:245-248.

2. Beutler E. The designation of mutations. Am J Hum Genet. 1993:53:783-785.

3. Karki R, Pandya D, Elston RC, Ferlini C. Defining "mutation" and "polymorphism" in the era of personal genomics. BMC Med Genomics. 2015;15;8:37.

4. Lacko M, Braakhuis BJ, Sturgis EM, Boedeker CC, Suárez C, Rinaldo A, et al. Genetic susceptibility to head and neck squamous cell carcinoma. Int J Radiat Oncol Biol Phys. 2014;89:38-48.

5. Hino O, Kobayashi T. Mourning Dr. Alfred G. Knudson: the two-hit hypothesis, tumor suppressor genes, and the tuberous sclerosis complex. Cancer Sci. 2017;108:5-11.

6. Famooto A, Almujtaba M, Dareng E, Akarolo-Anthony S, Ogbonna C, Offiong R, et al. RPS19 and TYMS SNPs and Prevalent high risk human papilloma virus infection in Nigerian women. PLoS One. 2013:27;8:e66930.

7. Kenney AD, Dowdle JA, Bozzacco L, McMichael TM, St Gelais C, Panfil AR, et al. Human genetic determinants of viral diseases. Annu Rev Genet. 2017:51:241-263.

8. Tandon N, Srivastava AN, Fatima N, Raza ST, Kumar V. p53 codon 72 gene polymorphism studies and p53 expression by immunohistochemistry in oral lesions as risk factor for malignancy. Int J Appl Basic Med Res. 2017;7:243-246.

9. Saleem S, Azhar A, Hameed A, Khan MA, Abbasi ZA, Qureshi NR, et al. P53 (Pro72Arg) polymorphism associated with the risk of oral squamous cell carcinoma in gutka, niswar and manpuri addicted patients of Pakistan. Oral Oncol. 2013;49:818-823.

10. Murali A, Nalinakumari KR, Thomas S, Kannan S. Association of single nucleotide polymorphisms in cell cycle regulatory genes with oral cancer susceptibility. Br J Oral Maxillofac Surg. 2014;52:652-658.

11. Sun Y, Yu W, Sturgis EM, Peng W, Lei D, Song X, et al. Site disparities in apoptotic variants as predictors of risk for second primary malignancy in patients with squamous cell carcinoma of the head and neck. BMC Cancer. 2016;16:70

12. Jin L, Sturgis EM, Zhang Y, Huang Z, Song X, Li C, et al. Association of tumor necrosis factor-alpha promoter variants with risk of HPV-associated oral squamous cell carcinoma. Mol Cancer. 2013;12:80.

13. Hsu HJ, Yang YH, Shieh TY, Chen CH, Kao YH, Yang CF, et al. TGF-b1 and IL-10 single nucleotide polymorphisms as risk factors for oral cancer in Taiwanese. Kaohsiung J Med Sci. 2015;31:123-129.

14. Antonsson A, Law MH, Neale RE, Coman WB, Pryor DI, Whiteman DC, et al. Variants of EVER1 and EVER2 (TMC6 and TMC8) and human papillomavirus status in patients with mucosal squamous cell carcinoma of the head and neck. Cancer Causes Control. 2016;27:809-815.g 